PROCEEDINGS OF THE

AMERICAN MATHEMATICAL SOCIETY

Volume 138, Number 4, April 2010, Pages 1387-1393

S 0002-9939(09)10146-6

Article electronically published on October 28, 2009

\title{
ASYMPTOTICALLY CONSTANT LINEAR SYSTEMS
}

\author{
HORST BEHNCKE
}

(Communicated by Yingfei Yi)

\begin{abstract}
The asymptotic integration of a linear system of differential equations $y^{\prime}(t)=(A(t)+R(t)) y(t)$ on the half line is investigated when $A$ is almost constant with distinct eigenvalues. The difference equation analogue of this equation is also considered.
\end{abstract}

\section{INTRODUCTION}

We are considering systems of differential equations of the form

$$
y^{\prime}(t)=\left(A(t)+R_{1}(t)\right) y(t) \quad \text { on }[0, \infty) .
$$

The complex valued matrix $A(t)$ is assumed to be smooth and to converge to a limiting matrix $A$ with distinct eigenvalues. The perturbing matrix $R_{1}$ is supposed to be integrable, $R_{1} \in \mathcal{L}^{1}$. If $A(t)$ is assumed to be $k$-times differentiable, $A^{(l)} \in$ $\mathcal{L}_{0}^{k / l}, 1 \leq l \leq k$, will do, this system may be transformed into [1, 2, 6]

$$
\begin{aligned}
& u^{\prime}(t)=(\wedge(t)+R(t)) u(t) \quad \text { with } \wedge=\operatorname{diag}\left(\lambda_{1}(t), \ldots, \lambda_{n}(t)\right), \\
& \lambda_{i}(t) \rightarrow \lambda_{i 0} \quad i=1, \ldots, n \text { and } R \in \mathcal{L}^{1}
\end{aligned}
$$

by repeated diagonalization. Here $\mathcal{L}_{0}^{p}$ stands for all $\mathcal{L}^{p}$-functions vanishing at infinity. A system of the form (1.1) will be called a Levinson system, in honour of N. Levinson, who studied such equations with $A^{\prime} \in \mathcal{L}^{1}$.

Levinson's Theorem is arguably the key result in asymptotic integration of linear differential systems. In a slightly modernized form it states [6]:

Theorem L: Let $\wedge(x)$ be an $n$ by $n$ diagonal matrix, $\wedge=\operatorname{diag}\left(\lambda_{1}(x), \ldots, \lambda_{n}(x)\right)$, satisfying the dichotomy conditions

$$
\int_{t}^{x} \operatorname{Re}\left(\lambda_{i}(s)-\lambda_{j}(s)\right) d s \leq K_{1} \quad \text { or } \int_{t}^{x} \operatorname{Re}\left(\lambda_{i}(s)-\lambda_{j}(s)\right) d s \geq K_{2}
$$

for constants $K_{1}$ and $K_{2}$ and $0 \leq t \leq x$. Assume the complex valued matrix $R(x)=\left(R_{i j}(x)\right)$ is integrable, i.e.

$$
\int_{a}^{\infty} \sum_{i, j=1}^{n}\left|R_{i j}(x)\right| d x<\infty .
$$

Then the system (1.1) has $n$ solutions

$$
u_{k}(x)=\left(e_{k}+r_{k}(x)\right) \exp \left(\int_{a}^{x} \lambda_{k}(t) \mathrm{dt}\right) \quad k=1, \ldots, n,
$$

Received by the editors May 13, 2009, and, in revised form, July 31, 2009.

2000 Mathematics Subject Classification. Primary 34E10.

(c)2009 American Mathematical Society $1387^{\text {Reverts to public domain } 28 \text { years from publication }}$ 
where the $e_{k}$ are the unit vectors in $\mathbb{C}^{n}$ and where $r_{k}=o(1)$.

Thus Levinson's Theorem states that the solutions (1.4) of the perturbed equation (1.1) behave almost like the solutions of the unperturbed equation $u^{\prime}=\wedge u$, if the relative growth of these solutions can be controlled for large $x(1.2)$ and if the perturbation is small (1.3).

Viewed in this way, as a perturbation result, Levinson's Theorem has been extended in various ways by weakening or strengthening the dichotomy condition (1.2) and strengthening or weakening the conditions on the perturbing term [5]. Most of these extensions were based on transformations of the system under investigation into Levinson's form. A new approach to such results on asymptotic integration has recently been initiated by S. Bodine [6]. For spectral analysis, parameter dependent versions of Levinson's Theorem were shown by Remling and the author.

Critical for the asymptotic integration of Levinson systems is the dichotomy condition [6, 8, which allows a uniform comparison of two solutions of the unperturbed equation. Levinson's Theorem formulation has two defects. First of all, better estimates for the remainder terms $r_{k}$ are needed. This has been addressed in [3, 4. Second, one might want to know how much of Levinson's classical result may be salvaged if the dichotomy condition fails. Our main goal is to weaken (1.2) and reduce (1.4) to sharp estimates on the growth.

\section{LeVINSON SYSTEMS WiThout Dichotomy}

Our main result is:

Theorem 2.1. Consider the system (1.1), and assume $\lambda_{i}(t)=\lambda_{i 0}+\lambda_{i 1}(t)$ with $\lambda_{i 1}=o(1)$ for $i=1, \ldots, n$. Sort the eigenvalues into classes $\mathcal{C}_{1}, \ldots, \mathcal{C}_{k}$ so that

i) if $\lambda_{i} \in \mathcal{C}_{l}$, then $\operatorname{Re} \lambda_{i 0}=\alpha_{l}$;

ii) if $\lambda_{i} \in \mathcal{C}_{l}, \lambda_{j} \in \mathcal{C}_{m}, l \neq m$, then $\left|\operatorname{Re}\left(\lambda_{i 0}-\lambda_{j 0}\right)\right| \geq \delta>0, l, m=l, \ldots, k$.

Now let $m_{l \pm}=\max _{\lambda_{i} \in \mathcal{C}_{l}}\left(\operatorname{Re}\left(\lambda_{i},(t)-\alpha_{l}\right)_{ \pm}\right.$, where $f_{( \pm)}$denotes the positive (negative) part of $f, f=f_{+}-f_{-}$. Let $\left|\mathcal{C}_{l}\right|$ denote the number of elements in $\mathcal{C}_{l}$. Then (1.1) has $\left|\mathcal{C}_{l}\right|$ independent solutions $u$ associated to $\mathcal{C}_{l}$ satisfying

$$
K_{1} \exp \left(\alpha_{l} x-\int_{a}^{x} m_{l-}(t) d t\right) \leq\|u(x)\| \leq K_{2} \exp \left(\alpha_{l} x+\int_{a}^{x} m_{l+}(t) d t\right)
$$

with constant $K_{1}, K_{2}$.

Proof. a) The upper bound.

It suffices to show the result for $\mathcal{C}_{1}=\left\{\lambda_{1}, \ldots, \lambda_{l}\right\}$ only. Shift the eigenvalues globally by $-\left(\alpha_{1}+m_{1+}\right)$ [6, (1.4.1)]. This results in $\operatorname{Re} \lambda_{i 1} \leq 0, i=1, \ldots, l$. Choose $a$ so large that

$$
\sum_{i, j} \int_{a}^{\infty}\left|R_{i j}(t)\right| \mathrm{dt}<\epsilon .
$$

$\epsilon>0$ will be fixed later. Let $P_{1}$ be the projection onto the coordinate vectors $e_{1}, \ldots, e_{l}$ and all $e_{j}$ for which $\operatorname{Re} \lambda_{j}(x) \leq 0$. Thus $P_{1}$ is the projection on the space of all decaying solutions of the modified unperturbed equation. The existence and corresponding estimates follow from the dichotomy condition (1.2). Moreover, let $\phi(x)=\operatorname{diag}\left(\exp \left(\int_{a}^{x} \lambda_{i}(t) \mathrm{dt}\right)\right)$ be the fundamental matrix of the unperturbed system. 
Then for $m=1, \ldots, l$ any solution of the integral equation

$$
\begin{aligned}
& v(x)=\phi(x) e_{m}+\phi(x) \int_{a}^{x} P_{1} \phi^{-1}(t) R(t) v(t) \\
& -\phi(x) \int_{x}^{\infty}\left(1-P_{1}\right) \phi^{-1}(t) R(t) v(t) \mathrm{dt}
\end{aligned}
$$

solves (1.1). By assumption there is a constant $K$ so that

$$
\begin{gathered}
\left\|\phi(x) P_{1} \phi^{-1}(t)\right\| \leq K, a \leq t \leq x ; \\
\left\|\phi(x)\left(1-P_{1}\right) \phi^{-1}(t)\right\| \leq K, a \leq x \leq t .
\end{gathered}
$$

Following the proof of Levinson's Theorem in [6] we solve (2.2) by iteration with $v_{0}(x)=\phi(x) e_{m}$ as the starting vector:

$$
\begin{aligned}
& v_{r+1}(x)=\phi(x) e_{m}+\phi(x) \int_{a}^{x} P_{1} \phi^{-1}(t) R(t) v_{r}(t) \mathrm{dt} \\
& -\phi(x) \int_{x}^{\infty}\left(1-P_{1}\right) \phi^{-1}(t) R(t) v_{r}(t) \mathrm{dt} .
\end{aligned}
$$

If $K \epsilon<\frac{1}{4}$, it follows by induction that $\left\|v_{r}(x)\right\| \leq 2$ and thus

$$
\left\|v_{r+1}(x)-v_{r}(x)\right\| \leq 2 K \epsilon|| v_{r}(x)-v_{r-1}(x)\left\|\leq \cdots \leq(2 K \epsilon)^{r}\right\| v_{1}(x)-v_{0}(x) \| .
$$

Thus the sequence $\left(v_{r}(x)\right)$ converges uniformly on $[a, \infty)$ to a solution $v$ of (1.1) with $\left\|v(x)-v_{0}(x)\right\| \leq 2 K \epsilon(1-2 K \epsilon)^{-1}$. In particular if $K \epsilon$ is small enough, the $l$ solutions starting with $e_{1}, \ldots, e_{l}$ are independent. The boundedness of these solutions gives the upper bound because of the shift in the exponential by $-\alpha-m_{1+}$.

b) The lower bound.

In order to show the lower bound, shift the exponents globally by $-\alpha+m_{1-}$ if necessary. In this case we may assume $\alpha_{1}=0$ and $\operatorname{Re} \lambda_{i 1}(x) \geq 0, i=1, \ldots, l$. Arguing indirectly, assume one of the solutions $v$ constructed above, with starting vector $\phi e_{m}$, satisfies $v(x)=o(1)$. Now let $P_{2}$ be the projections onto all coordinate vectors $e_{i}$ with $\operatorname{Re} \lambda_{i}(x) \leq 0$ and $i>l$. Note that in this case $\left(1-P_{2}\right) e_{i}=e_{i}, i=$ $1, \ldots, l$. Consider now

$$
w(x)=v(x)-\int_{a}^{x} \phi(x) P_{2} \phi^{-1} R(t) v(t) \mathrm{dt}+\int_{x}^{\infty} \phi(x)\left(1-P_{2}\right) \phi^{-1}(t) R(t) v(t) \mathrm{dt} .
$$

Then $w^{\prime}=\wedge w$, and arguing as in 2], we see $(w(x)-v(x))=o(1)$. By choosing $a$ large enough, $\|w(a)-v(a)\|$ can be made arbitrarily small. This, however, contradicts $w^{\prime}=\wedge w$, because then $\|w(x)\| \geq C \cdot \exp \int_{a}^{x} \operatorname{Re} \lambda_{m}(t) \mathrm{dt}$.

Remark 2.1. This theorem may not be optimal in all cases, e.g. when the dichotomy condition also holds within some classes.

\section{EXTENSIONS}

The theorem has a number of simple extensions.

1. The convergence condition in (1.1) need not hold. Only condition (ii) in the theorem is relevant. In this form these results arise in connection with the stability of exponential dichotomy [7, where more general perturbations can be allowed. The estimates of the theorem are sharper, however. In addition, such inequalities need to hold only modulo integrable terms. 
2. In the theorem a different and finer scale may be used. For this let $h$ be a positive nonintegrable function converging to 0 as $t$ goes to infinity. Assume the classes satisfy:

i) if $\lambda_{1}, \lambda_{j} \in \mathcal{C}_{k}$, then $\operatorname{Re}\left(\lambda_{i}(x)-\lambda_{j}(x)\right)=o(h)$; and

ii) if $\lambda_{i} \in \mathcal{C}_{l}, \lambda_{j} \in \mathcal{C}_{m}, l \neq m$, then $\left|\operatorname{Re}\left(\lambda_{i}(x)-\lambda_{j}(x)\right)\right| \geq h(x)$.

In this case the theorem would give the estimates.

$$
K_{1} \exp \int_{a}^{x} m_{k-}(t) \mathrm{dt} \leq\|u(x)\| \leq K_{2} \int_{a}^{x} m_{k+}(t) \mathrm{dt}
$$

where $m_{k+}=\max _{\lambda_{i} \in \mathcal{C}_{k}}\left(\operatorname{Re} \lambda_{j}(x)\right), m_{k-}=\min \operatorname{Re} \lambda_{j}(x)$.

3. Even more general is the formation of order classes $\mathcal{C}_{l}$. In this case one would demand:

if $\lambda_{i} \in \mathcal{C}_{l}, \lambda_{j} \in \mathcal{C}_{m}, l \neq m$, then either $\operatorname{Re} \lambda_{i} \geq \operatorname{Re} \lambda_{j}$ or $\operatorname{Re} \lambda_{i} \leq \operatorname{Re} \lambda_{j}$.

In both cases these relations need to hold only modulo $\mathcal{L}^{1}$.

4. The theorem will also be valid if the interclass perturbing terms are only assumed to be in $\mathcal{L}^{2}$. In this case one writes $R=R_{1}+R_{2}$, where $R_{1}$ consists of the block matrix of perturbing terms associated to various classes, while $R_{2}$ consists of the interblock parts. Thus $R_{1 i j}=0$ if $\lambda_{i} \in \mathcal{C}_{l}, \lambda_{j} \in \mathcal{C}_{m}$ and $l \neq m$. To $R_{2} \in \mathcal{L}^{2}$ apply a $(1+Q)$-transformation [1, 2, [5, transforming $R_{2}$ into the integrable term $R_{2} Q \in \mathcal{L}^{1}$. This would extend the Hartman-Wintner result. $R \in \mathcal{L}^{p}$ with $p>2$ could lead to uncontrollable terms within the blocks, however. Nonetheless one or several $(1+Q)$ transformations will be useful in most cases where $R \in \mathcal{L}^{p}$.

\section{Applications}

In applications of asymptotic integration to the deficiency index or spectral theory, one is mostly interested in the fact whether a solution is square integrable or not $[1,2,6$. In this case the dichotomy condition between exponents $\lambda$ with negative real part are not needed, and the analysis can concentrate on the exponents with "small" real part. In order to make this more precise, consider the results in [6], sect. 3.10, and [1, 2] applied to an even order differential operator of the form

$$
L y=w^{-1}\left\{\sum_{k=0}^{n}(-1)^{k}\left(p_{k} y^{(k)}\right)^{(k)}-i \sum_{j=1}^{n}(-1)^{j}\left(\left(q_{j} y^{(j)}\right)^{(j-1)}+\left(q_{j} y^{(j-1)}\right)^{(j)}\right)\right\}
$$

[1], (1.1), 5], (3.11). The coefficients are supposed to be real valued and twice differentiable [1, (1.2), with their derivatives satisfying some regularity conditions [1. (2.15), (2.20), (3.3), (3.5)], or [5, (3.2.4), (3.2.5), (3.2.6)]. For unbounded coefficients these conditions will have to be modified. With this, one can form the quasiderivatives of $y$ based on (4.1) and write $L y=z y$ in systems form [1], (2.3). With two diagonalizations this system can be transformed into a parameter dependent Levinson form. In this case the smoothness and decay conditions of the coefficients guarantee $R \in \mathcal{L}^{1}$. The exponentials $\lambda_{j}$ are just the roots of the characteristics polynomial $\mathfrak{P}$, [6, (3.23)], [1, (2.8)]. However, it is more advantageous to employ the Fourier polynomial. This is obtained by replacing $\lambda$ by $-i \lambda$. It has the form

$$
\mathfrak{P}_{F}(x, \lambda, z)=\sum_{k=0}^{n} p_{k}(x) \lambda^{2 k}+2 \sum_{j=1}^{n} q_{j}(x) \lambda^{2 j-1}-z w
$$


For real spectral values it is a polynomial with real coefficients. Now assume the coefficients are almost constant [6], (3.2.2), [1], (3.1), i.e. $p_{k}(x) \rightarrow c_{k}, q_{j}(x) \rightarrow$ $d_{j}, w(x) \rightarrow 1, c_{n}=1$. Then the conditions of the theorem above are satisfied where the exponents are $(i)$-times the roots of the limiting Fourier polynomial

$$
Q_{F}(\lambda, z)=\sum c_{k} \lambda^{2 k}+\sum d_{j} \lambda^{2 j-1}-z .
$$

Assume the roots of $Q_{F}$ are for $z_{0} \in \mathbb{R}, \alpha_{1} \pm i \beta, \ldots, \alpha_{r} \pm i \beta_{r}, \gamma_{2 r+1}, \ldots, \gamma_{2 n}$ with $\alpha_{j}, \beta_{j}, \gamma_{l} \in \mathbb{R}$. Then the nonreal eigenvalues lead to $r$ square integrable solutions, which decay exponentially, because of the theorem. This holds regardless of the dichotomy conditions. For the real eigenvalues $\gamma_{l}$ we have by the implicit function theorem

$$
\gamma_{l}(z) \approx \gamma_{l}\left(z_{0}\right)+\left(\partial_{\lambda} Q\left(\gamma_{l}\right)\right)^{-1}\left(z-z_{0}\right) \quad \text { for }\left|z-z_{0}\right| \text { small. }
$$

Thus the dichotomy condition holds if

$$
\partial_{\lambda} Q\left(\gamma_{l}\right) \neq \partial_{\lambda} Q\left(\gamma_{m}\right), \quad l \neq m
$$

because $\partial_{\lambda} Q(y)$ is real. Then $\gamma_{l}=\gamma_{l}\left(z_{0}\right)$ will contribute to the deficiency index if $\partial_{\lambda} Q\left(\gamma_{l}\right)>0$ because the corresponding exponent is $i \gamma_{l}\left(z_{0}+i \eta\right) \approx i \gamma_{l}\left(z_{0}\right)-$ $\eta\left(\partial Q\left(\gamma_{l}\left(z_{0}\right)\right)\right)^{-1}$. The associated eigenfunctions, however, will lose their square integrability as $\eta \rightarrow 0+$ if the coefficients are bounded. Since the signs of $\partial_{\lambda} Q(\gamma)$ are evenly distributed, half of the $\gamma$ 's will lead to square integrable solutions for $\eta=\operatorname{Im} z>0$ and the complementary $\gamma^{\prime}$ s will lead to square integrable solutions in the lower half plane. This shows that for bounded coefficients $L$ is limit point, i.e. def $L=(n, n)$. If the coefficients are unbounded, some of the $\gamma$-eigenfuctions may stay square integrable as $\operatorname{Im} z \searrow 0$. In this case the deficiency index may be def $L=(n+d, n+d), 0 \leq d \leq n$, if $d$ solutions of $L y=z y$ stay square integrable. But in all cases it suffices to check the dichotomy conditions only for the real roots of $\mathfrak{P}_{F}$, for example in (3.11.5) in [6] or in (3.22) of [1]. These results extend easily to the situation where higher order smoothness but slower decay holds for the coefficients.

For spectral analysis one fixes $z_{o} \in \mathbb{R}, a>0$ and $\epsilon>0$ so that the characteristic Fourier polynomial has distinct roots for $x \geq a, z \in K=\left\{z \in \mathbb{C}|| z-z_{0} \mid \leq \epsilon\right.$, $\operatorname{Im} z \geq 0\}$. A slight modification in the construction of the terms $m_{ \pm}$in the proof of the theorem then shows that the eigenfunctions associated to eigenvalues with $\operatorname{Re} \lambda_{i 0}<0$ depend analytically on the spectral parameter. Subjecting them to the boundary condition shows that they generate at most discrete eigenvalues. Thus continuous spectrum will only arise from eigenfunctions which lose their square integrability as $\operatorname{Im} z \searrow 0$, and only for these will one have to check the dichotomy condition.

These considerations clearly extend to other classes of operators, e.g. odd order operator 2, Dirac-operators or matrix valued Sturm-Liouville operators. In fact they can be shown for general Hamiltonian systems with almost constant coefficients. This will be shown in a joint paper with Hinton, which critically uses these results.

\section{Difference equations}

Here we are concerned with a system of difference equations of the form

$$
x(t+1)=(A(t)+R(t)) x(t), \quad t \geq t_{0}, R \in l^{1},
$$


where $A(t)$ converges to a limiting matrix $A$ with distinct nonzero eigenvalues $\lambda_{i} \neq 0$. If $A(t)$ is sufficiently smooth, this equation may be transformed into the Levinson-Benzaid-Lutz form [4:

$$
u(t+1)=(\wedge(t)+R(t)) u(t), \quad \wedge(t)=\operatorname{diag}\left(\lambda_{1}(t), \ldots, \lambda_{k}(t)\right)
$$

with

$$
\sum_{t=t_{0}}^{\infty} \| R(t)||\left|\lambda_{i}(t)^{-1}\right|<\infty
$$

Let $h>0$ be a nonsummable, monotonic function in $\mathbb{N}$ and assume the eigenvalues $\lambda$ can be sorted into classes $\mathcal{C}_{1}, \ldots, \mathcal{C}_{2}$, so that

$$
\begin{aligned}
& \text { if } \lambda_{i}, \lambda_{j} \in \mathcal{C}_{k} \text {, then }\left(\left|\frac{\lambda_{i}}{\lambda_{j}}(t)\right|-1\right)=o(h) \\
& \text { if } \lambda_{i} \in \mathcal{C}_{k}, \lambda_{j} \in \mathcal{C}_{k^{\prime}}, k \neq k^{\prime} \text {, then }\left|\frac{\lambda_{i}}{\lambda_{i}}(t)\right| \leq 1-h(t) \text { or }\left|\frac{\lambda_{i}}{\lambda_{j}}(t)\right| \geq 1+h(t) .
\end{aligned}
$$

For each $\lambda$ write now $|\lambda(t)|=1+\mu(t)$ with $\mu_{+}=\max (0, \mu)$ and $\mu_{-}=\min (0, \mu)$. Then define for class $k$

$$
a_{k}(t)=\max _{\lambda \in \mathcal{C}_{k}} \mu(t)_{+} \text {and } b_{k}(t)=\max _{\lambda \in \mathcal{C}_{k}} \mu(t)_{-} .
$$

With this we can now formulate the difference analogue of Theorem 2.1.

Theorem 5.1. Consider the system (5.2) with (5.3) and assume the eigenvalue $\lambda_{i}$ can be sorted into classes as in (5.4). Then associated to $\mathcal{C}_{k}$ there are $\left|\mathcal{C}_{k}\right|$ solutions $u$ of (5.2) satisfying

$$
K_{1} \Pi_{t=k_{0}}^{t-1}\left(1-b_{k}(t)\right) \leq\|u(t)\| \leq K_{2} \Pi_{t=t_{0}}^{t-1}\left(1+a_{k}(t)\right) .
$$

The proof will follow the same strategy as in Theorem 2.1 with the obvious modifications of course.

Proof. Again we may restrict the analysis to $\mathcal{C}_{1}=\left\{\lambda_{1}, \ldots, \lambda_{l}\right\}$. Multiply all eigenvalues by $\left(1+a_{1}(t)\right)^{-1}$ and replace $R(t)\left(1+m_{1}(t)\right)^{-1}$ by $R$, (2.5). Let $P_{1}$ be the projection onto all coordinate vectors $e_{i}$ for which $\left|\lambda_{j}(t)\right| \leq 1$. Let $\phi(t)=\operatorname{diag}\left(\Pi_{t=t_{0}}^{t-1} \lambda_{i}(t)\right)$ be the fundamental matrix for the unperturbed system. Then, for $1 \leq m \leq l$, any solution of the equation

$$
v(t)=e_{m} \phi(t)+\sum_{s=t_{0}}^{t-1} \phi(t) P_{1} \phi^{-1}(s) R(s) v(s)-\sum_{s=t}^{\infty} \phi(t)\left(1-P_{1}\right) \phi^{-1}(s) R(s) v(s)
$$

satisfies the difference equation with $v\left(t_{0}\right)=e_{m}$. If one chooses $t_{0}$ large enough, this equation can be solved by iteration and all solutions with different starting vector $e_{m}, 1 \leq m \leq l$, are independent and bounded. To obtain the lower bound, proceed just as above. Then $\sum\left(1-b_{k}(t)\right)^{-1}\|R(t)\|<\infty$ follows by induction on $n$. Of course the remarks for differential systems apply similarly in this case too as regards the extensions and applications. 


\section{REFERENCES}

[1] H. Behncke: Spectral Theory of Higher Order Differential Operators. Proc. London Math. Soc. (3), 92 (2006), 139-160. MR2192387(2007b:47109)

[2] H. Behncke, D.B. Hinton: Eigenfunctions, Deficiency Indices and Spectra of Odd-Order Differential Operators, Proc. London Math. Soc. (3), 97 (2008), 425-449. MR 2439668(2009g:34216)

[3] H. Behncke: The Remainder in Asymptotic Integration, Proc. Amer. Math. Soc., 136 (2008), 3231-3238. MR2407088 (2009c:34117)

[4] Z. Benzaid, D. A. Lutz: Asymptotic Representation of Solutions of Perturbed Systems of Linear Difference Equations, Stud. Appl. Math., 77 (1987), 195-221. MR1002291 (90f:39003)

[5] S. Bodine: A Dynamical Systems Result on Asymptotic Integration of Linear Differential Systems, J. Diff. Eqn., 187 (2003), 1-22. MR.1946543 (2003m:34109)

[6] M. S. P. Eastham: The Asymptotic Solution of Linear Differential Systems, London Math. Soc. Monographs, The Clarendon Press, Oxford University Press, New York, 1989. MR 1006434 (91d:34001)

[7] N. Ju, S. Wiggins: On Roughness of Exponential Dichotomy, J. Math. Anal. Appl., 262 (2001), 39-49. MR1857213 (2002g:34100)

[8] N. Levinson: The Asymptotic Nature of Solutions of Linear Systems of Differential Equations, Duke Math. J., 15 (1948), 111-126. MR0024538(9:509h)

Fachbereich Mathematik/Informatik, Universität OsnabrüCk, 49069 OsnabrüCK, GerMANY 